Check for updates

Cite this: RSC Adv., 2017, 7, 34466

Received 18th May 2017

Accepted 26th June 2017

DOI: 10.1039/c7ra05609e

rsc.li/rsc-advances

\section{Modification of the intermediate band and thermoelectric properties in Se-doped $\mathrm{CoSbS}_{1-x} \mathrm{Se}_{x}$ compounds $\uparrow^{\dagger}$}

\author{
Yonghui You, ${ }^{a}$ Xianli Su, ${ }^{\star a}$ Wei Liu, ${ }^{a}$ Yonggao Yan, ${ }^{a}$ Tiezheng Hu, ${ }^{a}$ Ctirad Uher ${ }^{b}$ \\ and Xinfeng Tang (D) *a
}

\begin{abstract}
Paracostibite (CoSbS), a naturally occurring mineral composed of earth abundant elements, is a newly developed and environmentally friendly thermoelectric material for medium temperature power generation applications, and has attracted considerable attention. In this work, in order to study the influence of Se doping on the site of $\mathrm{S}, \mathrm{CoSbS}_{1-x} \mathrm{Se}_{x}(0 \leq x \leq 0.09)$ compounds were synthesized by vacuum melting and annealing and compacted by spark plasma sintering. Alloying $S$ with Se decreased the band gap and the impurity activation energy. Moreover, Se substitution on the site of $\mathrm{S}$ not only improved the electronic transport properties, but it also dramatically suppressed the thermal conductivity. The maximum power factor as high as $1.1 \times 10^{-3} \mathrm{Wm}^{-1} \mathrm{~K}^{-2}$ was achieved in $\mathrm{CoSbS}_{0.99} \mathrm{Se}_{0.01}$ at $900 \mathrm{~K}$. Due to the improved power factor and the decreased thermal conductivity, the $Z T$ reached 0.26 , exceeding the figure of merit of undoped CoSbS by about $37 \%$.
\end{abstract}

\section{Introduction}

Thermoelectric (TE) materials are able to convert heat into electricity by purely solid-state means and have drawn widespread attention in recent years. ${ }^{1}$ Generally, the thermoelectric performance is characterized by the dimensionless figure of merit $Z T=\alpha^{2} \sigma T /\left(\kappa_{\mathrm{L}}+\kappa_{\mathrm{c}}\right)$, where $\alpha$ is the Seebeck coefficient, $\sigma$ is the electrical conductivity, $\kappa_{\mathrm{L}}$ is the lattice thermal conductivity, $\kappa_{\mathrm{c}}$ is the electronic thermal conductivity and $T$ is the absolute temperature. ${ }^{2,3}$ An excellent thermoelectric material should simultaneously possess high electrical conductivity, large Seebeck coefficient and low thermal conductivity. However, the above mentioned transport parameters are mutually interdependent and it is a challenge to improve the overall $Z T$ value. So far, numerous investigations have focused on band structure modifications, ${ }^{4,5}$ trying to find new thermoelectric materials, creating nanometer-scale secondary phases in the matrix ${ }^{6-8}$ and making use of hierarchical architectures ${ }^{9-12}$ in order to improve the thermoelectric performance. Although these approaches have greatly improved thermoelectric properties of materials such as $\mathrm{Bi}_{2} \mathrm{Te}_{3}$ (ref. 13,14), bulk chalcogenides ${ }^{15,16}$ and other thermoelectrics, ${ }^{17,18}$ many of them are not suitable for largescale applications given the toxicity of their constituent

${ }^{a}$ State Key Laboratory of Advanced Technology for Materials Synthesis and Processing, Wuhan University of Technology, Wuhan 430070, China. E-mail: suxianli@whut.edu. cn; tangx@@whut.edu.cn

${ }^{b}$ Department of Physics, University of Michigan, Ann Arbor, MI 48109, USA

$\dagger$ Electronic supplementary information (ESI) available. See DOI: $10.1039 / \mathrm{c} 7 \mathrm{ra} 05609 \mathrm{e}$ elements, limited availability, complex synthesis processing and poor stability at high temperatures. Thus, it is important to develop new, efficient, inexpensive and environmentally benign thermoelectric materials. During the past few years, certain minerals consisting of transition metal chalcogenide compounds have attracted extensive attention for their potential as prospective TE materials. Such minerals are earth abundant, environmentally friendly, and several of them are narrow band gap semiconductors. ${ }^{19-24}$

Paracostibite (CoSbS) is one of such minerals, first found in Broken Hill, N.S.W., Australia and the Red Lake area of Ontario in Canada and reported on by Cabri et al. ${ }^{25}$ It crystallizes in the orthorhombic structure with the space group $61(P b c a)$, having a band gap around $0.75 \mathrm{eV},{ }^{26}$ and a relatively high Seebeck coefficient at medium temperatures. Unfortunately, the thermoelectric performance of the pristine CoSbS compound is poor due to its intrinsically low carrier concentration $\left(n \sim 10^{17}\right.$ $\mathrm{cm}^{-3}$ ). Many investigations have focused on devising a synthesis process to yield a high purity single phase CoSbS compound and on optimization of the carrier concentration through doping with $\mathrm{Ni}$ or Te to enhance its thermoelectric properties. ${ }^{26-31}$ However, a strong ionic bond between Co and S, having the origin in a large electronegativity difference between $\mathrm{S}$ and Co atoms, leads to the intrinsically low carrier mobility ( $\mu$ $\sim 4 \mathrm{~cm}^{2} \mathrm{~V}^{-1} \mathrm{~s}^{-1}$ ) and high thermal conductivity. This has hindered any significant improvement of the TE properties. Thus, decreasing the ionic character of the $\mathrm{Co}-\mathrm{S}$ bond and weakening the effect of deep impurity levels on the transport properties are crucial steps towards improving the TE performance of CoSbS-based compounds. 
In this work, we have synthesized a series of Se-doped $\operatorname{CoSbS}_{1-x} \mathrm{Se}_{x}(x=0-0.09)$ compounds by vacuum melting and annealing followed by SPS processing, and we evaluate their structural and transport properties.

\section{Experimental}

To avoid the influence of commonly found impurities (Fe, Ni, As) in mineral forms of paracostibite and to study its intrinsic properties, we synthesized pure CoSbS and investigated the effect of Se dopants on the structural and transport properties.

Appropriate amounts of cobalt (shot, 99.99\%), antimony (shot, 99.99\%), sulphur (pieces, 99.99\%), and Se (small chunks, 99.99\%) were weighed according to the stoichiometric composition of $\mathrm{CoSbS}_{1-x} \mathrm{Se}_{x}(x=0-0.09)$. The charge was loaded into a quartz tube and then sealed under vacuum. The tubes were placed in the furnace and heated up to $1273 \mathrm{~K}$ over a period of $10 \mathrm{~h}$. After soaking at $1273 \mathrm{~K}$ for $30 \mathrm{~h}$, the samples were quenched in supersaturated salt water and then annealed at 973 $\mathrm{K}$ for 3 days. Following cooling down to a room temperature, the obtained ingots were pulverized and compacted by the spark plasma sintering process with a pressure of $65 \mathrm{MPa}$ at $973 \mathrm{~K}$ for 8 minutes.

The phase composition of the samples was determined by powder X-ray diffraction (XRD, Empyrean, PANalytical) with $\mathrm{Cu}$ $\mathrm{K}_{\alpha}$ radiation. The chemical composition of the samples was characterized by electron probe microanalysis (EPMA, JXA-8230/ INCAX-ACT). The electrical conductivity and the Seebeck coefficient were measured simultaneously using the commercial equipment (ZEM-3, Ulvac Riko, Inc.) by the standard four-probe method under He atmosphere. The thermal diffusivity $\lambda$ was obtained by a laser flash technique on a Netzsch LFA-457 apparatus. The heat capacity $C_{\mathrm{p}}$ at constant pressure was measured by a comparison method with a DSC (TA Instrument Q20). The experimental density $d$ was evaluated by the Archimedes method. The thermal conductivity $\kappa$ was then calculated using the formula $\kappa=\lambda C_{\mathrm{p}} d$. All physical properties were measured in the temperature range of 300-900 K. Uncertainties in measurements of the electrical conductivity, Seebeck coefficient and thermal conductivity are estimated as $\pm 5 \%, \pm 2 \%$ and $\pm 5 \%$, respectively.

\section{Results and discussion}

\section{Phase composition and lattice constant}

Fig. 1(a) shows XRD patterns of the $\mathrm{CoSbS}_{1-x} \mathrm{Se}_{x}(x=0-0.09)$ compounds. The patterns show that all compounds are single phase materials with all peaks corresponding to the peaks of the CoSbS compound that crystallizes in the orthorhombic structure, space group 61 (Pbca). Even for the highest Se doping content $x=0.09$, there is no trace of any secondary phase within the XRD detection limit. Fig. 1(b) displays the lattice constants of the sintered samples as a function of the content of Se calculated by the Rietveld refinement method through a program called Fullproof. The lattice parameters increase linearly with the increasing Se content (except for the sample with $x=0.09$ ), mainly due to the larger covalent radius of Se
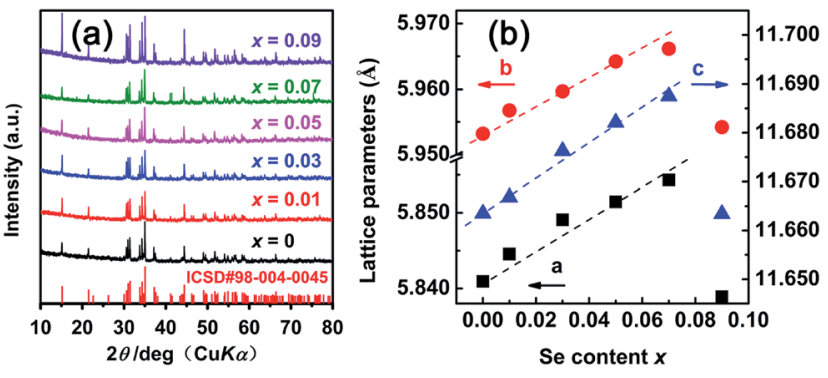

Fig. 1 (a) XRD patterns of the $\operatorname{CoSbS}_{1-x} \mathrm{Se}_{x}(0 \leq x \leq 0.09)$ compounds; (b) lattice parameters of $\mathrm{CoSbS}_{1-x} \mathrm{Se}_{x}(0 \leq x \leq 0.09)$ as a function of the content of Se.

(1.16 $\AA$ ) in comparison to that of $\mathrm{S}(1.02 \AA)$, which is consistent with the Vegard's law. For the sample with $x=0.09$, the lattice constants behave anomalously because of a small amount of the secondary phase present that was detected by means more sensitive than XRD (more about it below).

The Rietveld refinement was done on the XRD data of the $x=$ 0.01 compound (a slow scan with the step size $0.00656^{\circ}$ within the angular range of $\left.10^{\circ}-130^{\circ}\right)$. Fig. 2 displays the Rietveld refinement results and Table 1 lists the refined parameters with the quality factors $R_{\mathrm{wp}}, R_{\mathrm{p}}, \chi^{2}$ of $0.0382,0.0311$ and 1.151, respectively, proving the reliability of the refinement process. The above results verify that the Se atoms successfully enter the sites of $\mathrm{S}$ in the crystal lattice.

Fig. 3 displays FESEM images and back-scattering electron images (BSI) obtained on polished surfaces of sintered samples with $x=0.07$ and 0.09 . The FESEM results in Fig. 3(a) and (c) show that both samples are fully condensed. A few pits on the surface formed during the polishing process. No contrast is seen on the back-scattering electron image (BSI) of sample $x=$ 0.07 in Fig. 3(b), indicating that the structure is a homogeneous single phase material. In contrast, the sample with $x=0.09$ in Fig. 3(d) shows a trace amount of a secondary phase of

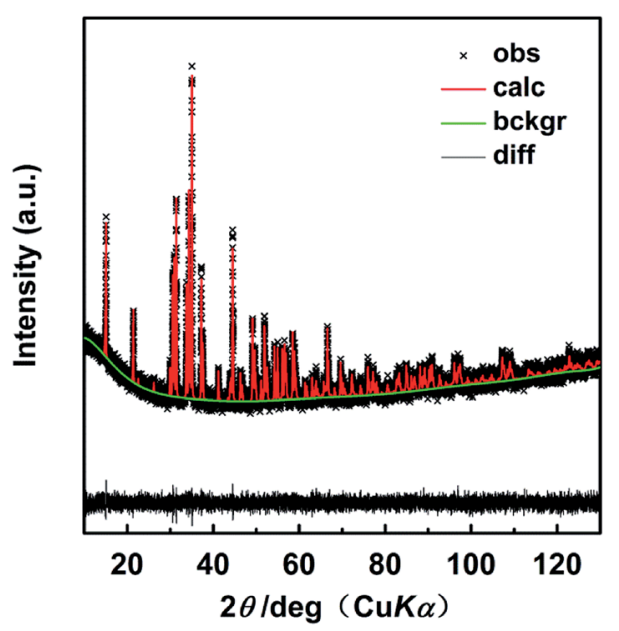

Fig. 2 Rietveld refinement of XRD pattern for $\mathrm{CoSbS}_{0.99} \mathrm{Se}_{0.01}$. The difference (black line) between the observed (black cross) and the calculated (red line) data is shown at the bottom. 
Table 1 Structure parameters and the Rietveld refined parameters for $\mathrm{CoSbS}_{0.99} \mathrm{Se}_{0.01}$. (a) Structure parameters for $\mathrm{CoSbS}_{0.99} \mathrm{Se}_{0.01}$. (b) Data collection and refined parameters by the Rietveld analysis for $\mathrm{CoSbS}_{0.99} \mathrm{Se}_{0.01}$

(a)

\begin{tabular}{lrlll}
\hline Atom & \multicolumn{1}{l}{$X$} & $Y$ & $Z$ & Occupancy \\
\hline Co & 0.021443 & 0.162091 & 0.384402 & 1 \\
Sb & 0.117535 & 0.049662 & 0.178686 & 1 \\
S & -0.124641 & 0.318852 & 0.063298 & 0.99 \\
Se & -0.124641 & 0.318852 & 0.063298 & 0.01 \\
\hline
\end{tabular}

(b)

\begin{tabular}{|c|c|c|c|}
\hline \multicolumn{2}{|c|}{ Data collection parameters } & \multicolumn{2}{|c|}{ Quality factors } \\
\hline X-ray radiation & $\mathrm{Cu} \mathrm{K}{ }_{\alpha}$ & $R_{\mathrm{wp}}$ & 0.0382 \\
\hline Monochromator & Graphite & & \\
\hline $2 \theta$, degree & $10-130$ & $R_{\mathrm{p}}$ & 0.0311 \\
\hline Step width, degree & 0.00656 & & \\
\hline Counts time, s & 43 & $\chi^{2}$ & 1.151 \\
\hline Temperature, $\mathrm{K}$ & 300 & & \\
\hline
\end{tabular}

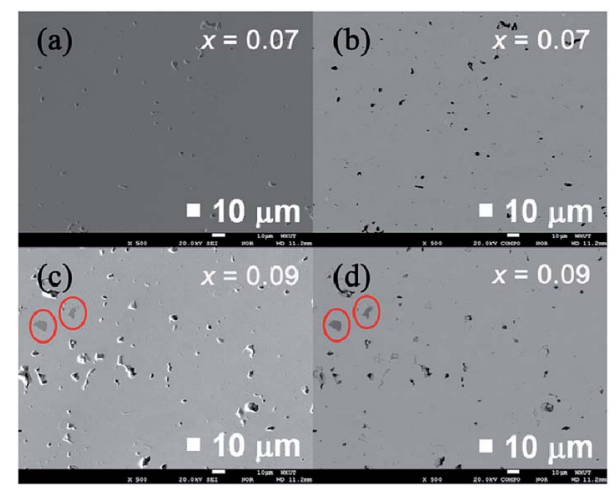

Fig. 3 (a, c) FESEM images and (b, d) back-scattering electron images of polished surfaces of sintered $\mathrm{CoSbS}_{1-x} \mathrm{Se}_{x}(x=0.07,0.09)$ compounds.

composition CoS, verified by EDS and consistent with the abnormal behaviour of the lattice constants in this highest Se content sample. Thus, we can conclude that $\operatorname{CoSbS}_{1-x} \mathrm{Se}_{x}$ compounds with the content of Se below 0.07 are single phase structures. As the content of Se exceeds 0.07, a secondary CoS phase is observed. Therefore, the solubility limit of Se in $\mathrm{CoSbS}_{1-x} \mathrm{Se}_{x}$ is around $x=0.07$.

\section{Electronic transport properties}

Fig. 4 displays the temperature dependence of electronic transport properties of $\mathrm{CoSbS}_{1-x} \mathrm{Se}_{x}$ compounds. Fig. 4(a) is the temperature dependent electrical conductivity $\sigma$. The data shows that, with the increasing content of Se, the electrical conductivity increases. This may be due to a smaller ionization energy of Se $\left(941.0 \mathrm{KJ} \mathrm{mol}^{-1}\right)$ compared to that of S $(999.6 \mathrm{KJ}$ $\mathrm{mol}^{-1}$ ). Since the ionization energy difference and the covalent radius difference between $\mathrm{Se}$ and $\mathrm{Sb}$ are smaller than that between $\mathrm{S}$ and $\mathrm{Sb}$, it is also possible that it is easier for Se to substitute on the site of $\mathrm{Sb}$, forming $\mathrm{Se}_{\mathrm{Sb}}$ antisite defects, which will move the Fermi level closer to the conduction band minimum by increasing the concentration of electrons. Moreover, Se substituting on the site of $S$ may weaken the ionic character of the Co-S bond in the compound, and thus improve the carrier mobility, which is beneficial to achieving a higher $\sigma .^{32-34}$

As Fig. 4(a) also documents, in the entire temperature range covered, $\sigma$ of all $\operatorname{CoSbS}_{1-x} \mathrm{Se}_{x}$ compounds increases with the increasing temperature in a roughly similar fashion, displaying a typical semiconducting behavior. In addition, a specific thermal activated behavior can be seen in Fig. 4(a) where, as the temperature increases the conductivity $\sigma$ increases more sharply. This likely arises from thermal excitations of electrons from the in-gap impurity states followed by across-the-gap electronic excitations at higher temperatures. To shed light on this issue, an Arrhenius plot of the resistivity $\rho$ vs. the inverse temperature is presented in Fig. 4(c). From the Arrhenius plot it follows that, at temperature below and above $700 \mathrm{~K}$, there are two distinct linear dependences between $\ln \rho$ and $1 / T$. This indicates the presence of two different transport mechanisms acting. At low and medium temperatures, the transport behavior is controlled by in-gap impurity state excitations, while at high temperatures, thermal excitations across the band gap are the dominant process. The electrical resistivity can be described $^{35-37}$ by the following model,

$$
\rho(T)=\rho_{1} T^{\left(-\frac{3}{2}\right)} \exp \frac{E_{\mathrm{a}}}{2 k_{\mathrm{B}} T}+\rho_{2} \exp \frac{E_{\mathrm{g}}}{2 k_{\mathrm{B}} T}
$$

where $\rho_{1}, \rho_{2}$ are temperature independent constants, $E_{\mathrm{a}}$ is the activation energy for impurity states and $E_{\mathrm{g}}$ is the band gap (both listed in Table 2). The first term represents excitations from in-gap impurity states and the second term accounts for the classic thermally activated excitations from the valence to the conduction band. From the results in Table 2 we note that, as the content $x$ of Se increases, the activation energy $E_{\mathrm{a}}$ and the band gap $E_{\mathrm{g}}$ of the compounds decrease (except for the $x=0.09$ sample). Depending on the content of Se, the activation energy $E_{\mathrm{a}}$ varies within the range of $0.14-0.26 \mathrm{eV}$, while the band gap $E_{\mathrm{g}}$ falls within the range of $0.63-0.69 \mathrm{eV}$. Thus, the presence of Se on sites of $\mathrm{S}$ reduces the activation energy of impurities and somewhat lowers the band gap, making it easier for electrons to enter the conduction band and improve the electrical conductivity.

Fig. 4(e) schematically visualizes the band structure of CoSbS. Impurity states in the band gap at energy $E_{\text {a }}$ below the conduction band edge start to be ionized at temperatures above $500 \mathrm{~K}$, generating electrons that sharply increase the electrical conductivity around that temperature. Thermal excitation of electrons across the band gap requires higher temperatures and, from the slope in Fig. 4(c), the band gap is about $0.69 \mathrm{eV}$.

Fig. 4(b) shows the temperature dependence of the Seebeck coefficient for $\mathrm{CoSbS}_{1-x} \mathrm{Se}_{x}$ compounds. The transport process in compounds with the Se content below 0.05 is dominated by positive charge carriers (holes) up to about $550 \mathrm{~K}$. At higher 

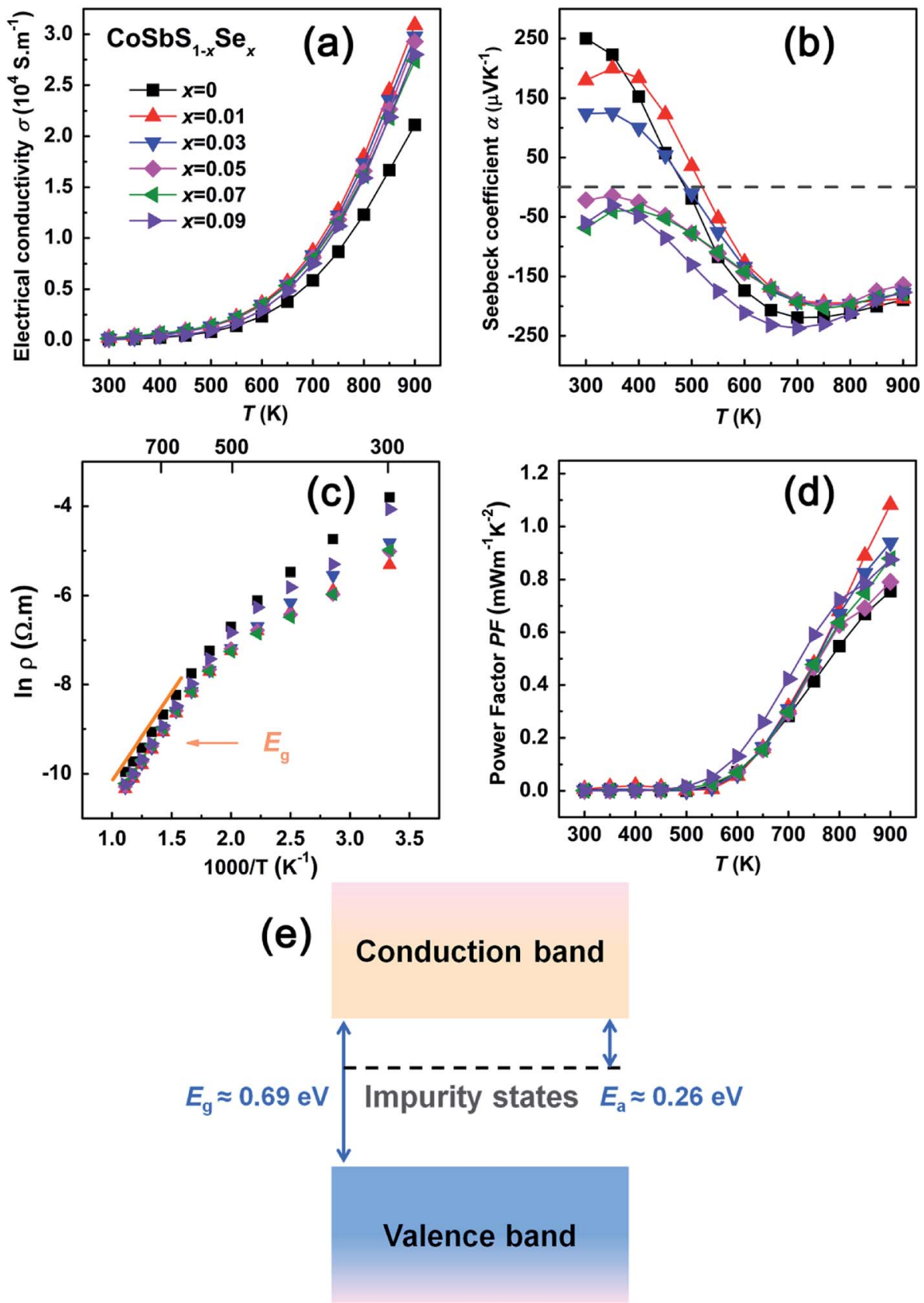

Fig. 4 Temperature dependence of electronic transport properties of $\mathrm{CoSbS}_{1-x} \mathrm{Se}_{x}(0 \leq x \leq 0.09)$ : (a) electrical conductivity, (b) Seebeck coefficient, (c) Arrhenius plot of the resistivity, and (d) power factor. (e) Schematic band structure for pure CoSbS with the position of the impurity states depicted with a dashed line.

temperatures, as the electrons thermally excited from the in-gap impurity states start to contribute to the transport, the Seebeck coefficient crosses over and becomes negative. In contrast, the compounds with the Se content exceeding 0.05 are n-type conductors at all temperatures. The diminishing magnitude of the Seebeck coefficient observed at temperatures above about $700 \mathrm{~K}$ is a consequence of intrinsic (across the gap) electron excitations. Typical Seebeck coefficients at $700 \mathrm{~K}$ reach values as much as $-200 \mu \mathrm{V} \mathrm{K} \mathrm{K}^{-1}$. Consequently, small and flat power factors of $\mathrm{CoSbS}_{1-x} \mathrm{Se}_{x}$ compounds at temperatures below $500 \mathrm{~K}$ turn rapidly into reasonably large power factors at high temperatures with values on the order of $1 \times 10^{-3} \mathrm{Wm}^{-1} \mathrm{~K}^{-2}$ at $900 \mathrm{~K}$.

\section{Thermal transport properties}

Fig. 5(a) and (b) display the temperature dependent total thermal conductivity $\kappa$ and the lattice thermal conductivity $\kappa_{\mathrm{L}}$ of $\operatorname{CoSbS}_{1-x} \operatorname{Se}_{x}$ compounds. The lattice thermal conductivity $\kappa_{\mathrm{L}}$ is derived using the Wiedemann-Franz law, $\kappa_{\mathrm{L}}=\kappa-L \sigma T$, where the Lorenz number $L$ is obtained from eqn (2)-(6), employing 
Table 2 Band gaps and activation energies of $\operatorname{CoSbS}_{1-x} \mathrm{Se}_{x}(0 \leq x \leq$ 0.09) compounds

\begin{tabular}{lcc}
\hline Sample & $E_{\mathrm{g}}$ & $E_{\mathrm{a}}$ \\
\hline$x=0$ & 0.69 & 0.26 \\
$x=0.01$ & 0.65 & 0.19 \\
$x=0.03$ & 0.65 & 0.19 \\
$x=0.05$ & 0.64 & 0.18 \\
$x=0.07$ & 0.63 & 0.14 \\
$x=0.09$ & 0.72 & 0.24
\end{tabular}

a simple parabolic band (SPB) model under the relaxation time approximation and assuming the dominance of acoustic phonon scattering (the scattering factor $r=-1 / 2$ ). ${ }^{38-40}$

$$
\begin{gathered}
\alpha= \pm \frac{k_{\mathrm{B}}}{e}\left[\eta_{\mathrm{F}}-\frac{(r+5 / 2) F_{r+3 / 2}\left(\eta_{\mathrm{F}}\right)}{(r+3 / 2) F_{r+1 / 2}\left(\eta_{\mathrm{F}}\right)}\right] \\
F_{\mathrm{i}}\left(\eta_{\mathrm{F}}\right)=\int_{0}^{\infty} \frac{x^{\mathrm{i}} \mathrm{d} x}{1+\exp \left(x-\eta_{\mathrm{F}}\right)} \\
\eta_{\mathrm{F}}=\frac{E_{\mathrm{F}}}{k_{\mathrm{B}} T} \\
L=\left(\frac{k_{\mathrm{B}}}{e}\right)^{2}\left[\frac{(r+7 / 2) F_{r+5 / 2}\left(\eta_{\mathrm{F}}\right)}{(r+3 / 2) F_{r+1 / 2}\left(\eta_{\mathrm{F}}\right)}-\delta^{2}\left(\eta_{\mathrm{F}}\right)\right]
\end{gathered}
$$

$$
\delta\left(\eta_{\mathrm{F}}\right)=\frac{(r+5 / 2) F_{r+3 / 2}\left(\eta_{\mathrm{F}}\right)}{(r+3 / 2) F_{r+1 / 2}\left(\eta_{\mathrm{F}}\right)}
$$

here, $\eta_{\mathrm{F}}, F_{\mathrm{i}}\left(\eta_{\mathrm{F}}\right), r$, and $k_{\mathrm{B}}$ represent the reduced Fermi level, the Fermi integral, the scattering factor, and the Boltzmann constant, respectively.

The total and the lattice thermal conductivities $\kappa$ and $\kappa_{\mathrm{L}}$ decrease smoothly and continuously with the increasing temperature, as shown in Fig. 5(a) and (b). Except for the compound with $x=0.09$, which contains secondary phases, the lattice thermal conductivity decreases as the content of Se increases.

The alloy scattering, i.e., the presence of Se on sites of S, has two aspects: it induces mass fluctuations (mass difference between $\mathrm{S}$ and Se), and it causes strain fluctuations (due to the size difference between atoms of S and Se). In order to separate the two contributions, we use the theory of Callaway. ${ }^{41-43}$ The calculated mass fluctuation scattering parameter $\Gamma_{\mathrm{M}}$ and the strain field fluctuation scattering parameter $\Gamma_{\mathrm{S}}$ are presented in Table S1 (in ESI $\dagger$ ) and the calculation process in detail is in the ESI. $\dagger$

The results in Table $\mathrm{S} 1 \uparrow$ show that, the scattering parameter $\Gamma=\Gamma_{\mathrm{M}}+\Gamma_{\mathrm{S}}$ increases with the increasing content of Se as both the mass defect and strain field fluctuation contributions increase as more Se enters the crystal lattice.

\section{The dimensionless figure of merit $Z T$}

Using the measured values of the electrical conductivity, Seebeck coefficient and thermal conductivity, the dimensionless
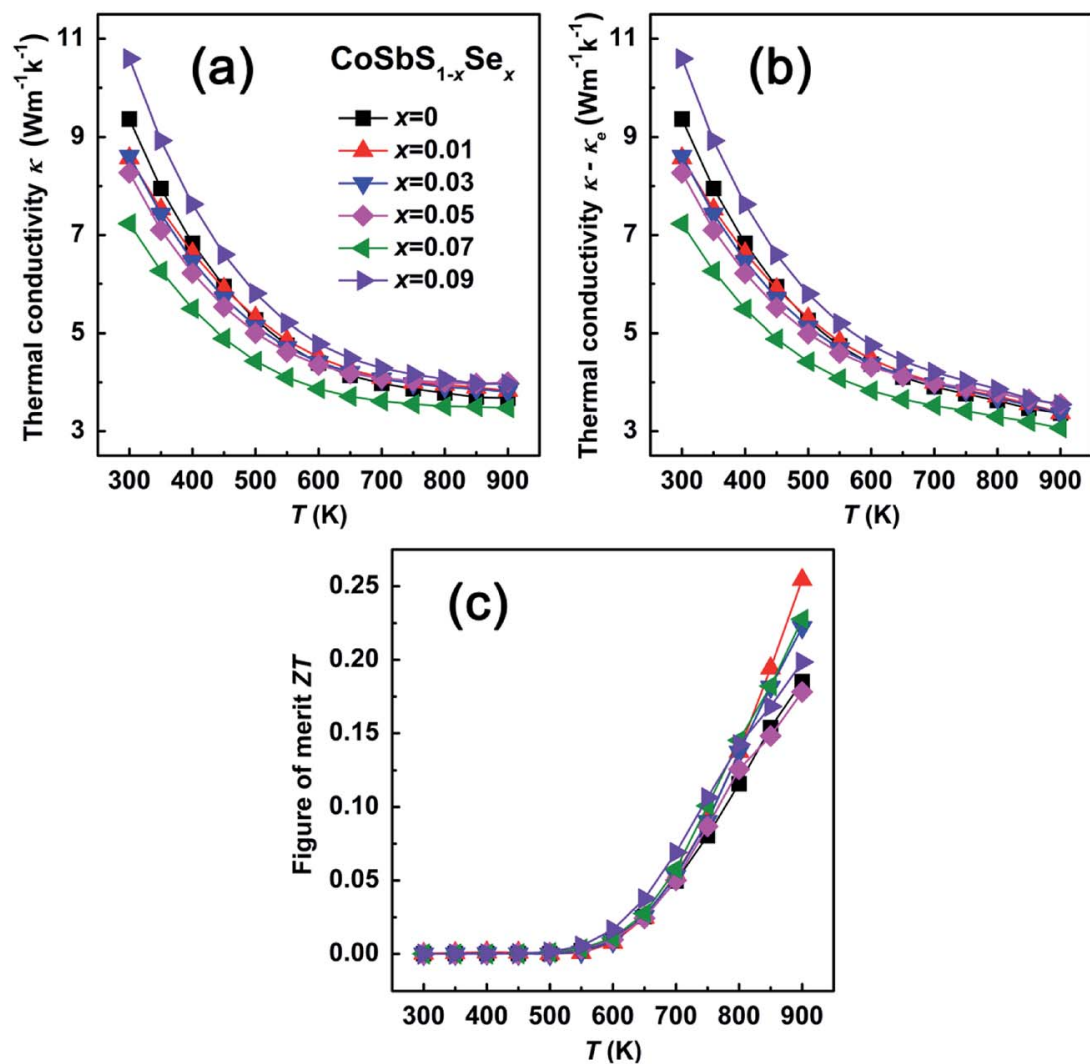

Fig. 5 Temperature dependence of (a) thermal conductivity, (b) lattice thermal conductivity and (c) $Z T$ of $\operatorname{CoSbS}_{1-x} \mathrm{Se}_{x}(0 \leq x \leq 0.09)$ compounds. 
thermoelectric figure of merit $Z T$ was calculated and its temperature dependence is presented in Fig. 5(c) for all $\operatorname{CoSbS}_{1-x} \operatorname{Se}_{x}(0 \leq x \leq 0.09)$ compounds. With the increasing temperature, the $Z T$ value increases rapidly, primarily because of ionization of the in-gap impurity levels and intrinsic excitations. Due to the enhanced power factor and the decreased thermal conductivity, the maximum $Z T$ of 0.26 is achieved at about $900 \mathrm{~K}$ in $\mathrm{CoSbS}_{0.99} \mathrm{Se}_{0.01}$. This is about $37 \%$ enhancement in the figure of merit compared to the undoped CoSbS compound at the same temperature.

\section{Conclusions}

A series of $\operatorname{CoSbS}_{1-x} \mathrm{Se}_{x}(0 \leq x \leq 0.09)$ compounds was prepared by vacuum melting and annealing followed by SPS processing. The solubility limit for Se on the site of $S$ is about 0.07. The electrical conductivity increases rapidly with the increasing temperature due to the ionization of impurity levels at around $500 \mathrm{~K}$ and due to intrinsic excitations setting in above $700 \mathrm{~K}$. The introduction of Se on the sites of $\mathrm{S}$ increases the electron density and the carrier mobility, leading to an increase in the electrical conductivity of Se-containing compounds. The Seebeck coefficient reflects a crossover from the p-type to n-type dominated carrier transport in low se content compounds, while compounds with the Se content equal or exceeding $x=$ 0.05 are invariably n-type conductors. The highest power factor of $1.1 \times 10^{-3} \mathrm{Wm}^{-1} \mathrm{~K}^{-2}$ at $900 \mathrm{~K}$ is measured in $\mathrm{CoSbS}_{0.99} \mathrm{Se}_{0.01}$. Owing to enhanced phonon scattering in compounds containing Se, the lattice thermal conductivity $\kappa_{\mathrm{L}}$ is markedly reduced and, together with the enhanced power factor, results in improved values of the dimensionless thermoelectric figure of merit. The highest value of $Z T=0.26$ at $900 \mathrm{~K}$ is achieved in $\mathrm{CoSbS}_{0.99} \mathrm{Se}_{0.01}$. This represents about $37 \%$ improvement over the figure of merit of pure CoSbS compound.

\section{Acknowledgements}

The authors wish to acknowledge support from the National Basic Research Program of China (973 program) under project 2013CB632502, the Natural Science Foundation of China (Grant No. 51402222, 51172174, 51521001, 51401153), and the 111 Project of China (Grant No. B07040).

\section{Notes and references}

1 G. J. Snyder and E. S. Toberer, Nat. Mater., 2008, 7, 105-114. 2 J. R. Sootsman, D. Y. Chung and M. G. Kanatzidis, Angew. Chem., Int. Ed., 2009, 48, 8616-8639.

3 A. Minnich, M. Dresselhaus, Z. Ren and G. Chen, Energy Environ. Sci., 2009, 2, 466-479.

4 W. Liu, X. Tan, K. Yin, H. Liu, X. Tang, J. Shi, Q. Zhang and C. Uher, Phys. Rev. Lett., 2012, 108, 166601.

5 Y. Pei, A. LaLonde, S. Iwanaga and G. J. Snyder, Energy Environ. Sci., 2011, 4, 2085-2089.

6 W. Xie, X. Tang, Y. Yan, Q. Zhang and T. M. Tritt, Appl. Phys. Lett., 2009, 94, 102111.
7 W. Xie, J. He, H. J. Kang, X. Tang, S. Zhu, M. Laver, S. Wang, J. R. Copley, C. M. Brown and Q. Zhang, Nano Lett., 2010, 10, 3283-3289.

8 B. Poudel, Q. Hao, Y. Ma, Y. Lan, A. J. Minnich, B. Yu, X. Yan, D. Wang, A. Muto and D. Vashaee, Science, 2008, 320, 634638.

9 G. J. Tan and M. G. Kanatzidis, in Materials Aspect of Thermoelectricity: All-Scale Architecture, ed. C. Uher, CRC press, Boca Raton, 2016, p. 125.

10 X. Su, P. Wei, H. Li, W. Liu, Y. Yan, P. Li, C. Su, C. Xie, W. Zhao, P. Zhai, Q. Zhang, X. Tang and C. Uher, Adv. Mater., 2017, 1602013.

11 L. Zhao, V. P. Dravid and M. G. Kanatzidis, Energy Environ. Sci., 2014, 7, 251-268.

12 Y. Zheng, Q. Zhang, X. Su, H. Xie, S. Shu, T. Chen, G. Tan, Y. Yan, X. Tang and C. Uher, Adv. Energy Mater., 2015, 5, 1401391.

13 S. K. Mishra, S. Satpathy and O. Jepsen, J. Phys.: Condens. Matter, 1997, 9, 461-470.

14 X. B. Zhao, X. Ji, Y. H. Zhang, T. J. Zhu, J. P. Tu and X. B. Zhang, Appl. Phys. Lett., 2005, 86, 062111.

15 A. P. Goncalves and C. Godart, Eur. Phys. J. B, 2014, 87, 1-29. 16 C. Han, Z. Li, G. Q. Lu and S. X. Dou, Nano Energy, 2015, 15, 193-204.

17 F. E. Akkad, B. Mansour and T. Hendeya, Mater. Res. Bull., 1981, 16, 535-539.

18 S. Ballikaya, H. Chi, J. R. Salvador and C. Uher, J. Mater. Chem., 2013, 1, 12478-12484.

19 X. Lu and D. T. Morelli, Phys. Chem. Chem. Phys., 2013, 15, 5762-5766.

20 X. Lu, D. T. Morelli, Y. Xia, F. Zhou, V. Ozolins, H. Chi, X. Zhou and C. Uher, Adv. Energy Mater., 2013, 3, 342-348.

21 E. J. Skoug, J. D. Cain and D. T. Morelli, J. Electron. Mater., 2012, 41, 1232-1236.

22 N. Tsujii, F. Meng, K. Tsuchiya, S. Maruyama and T. Mori, J. Electron. Mater., 2016, 45, 1642-1647.

23 Y. Zhang, E. J. Skoug, J. D. Cain, V. Ozolins, D. T. Morelli and C. Wolverton, Phys. Rev. B: Condens. Matter Mater. Phys., 2012, 85, 054306.

24 T. Wei, F. Li and J. Li, J. Electron. Mater., 2014, 43, 2229-2238.

25 L. J. Cabri, D. C. Harris and J. M. Stewart, Can. Mineral., 1970, 10, 232-246.

26 D. Parker, A. F. May, H. Wang, M. A. Mcguire, B. C. Sales and D. J. Singh, Phys. Rev. B: Condens. Matter Mater. Phys., 2013, 87, 045205.

27 Z. Liu, H. Geng, J. Shuai, Z. Wang, J. Mao, D. Wang, Q. Jie, W. Cai, J. Sui and Z. Ren, J. Mater. Chem. C, 2015, 3, 10442-10450.

28 R. Carlini, C. Artini, G. Borzone, R. Masini, G. Zanicchi and G. A. Costa, J. Therm. Anal. Calorim., 2010, 103, 23-27.

29 R. Chmielowski, S. Bhattacharya, W. Xie, D. Pere, S. Jacob, R. Stern, K. Moriya, A. Weidenkaff, G. K. H. Madsen and G. Dennler, J. Mater. Chem. C, 2016, 4, 3094-3100.

30 W. Yao, D. Yang, Y. Yan, K. Peng, H. Zhan, A. Liu, X. Lu, G. Wang and X. Zhou, ACS Appl. Mater. Interfaces, 2017, 9(12), 10595-10601. 
31 R. Chmielowski, S. Bhattacharya, S. Jacob, D. Pere, A. Jacob, K. Moriya, B. Delatouche, P. Roussel, G. Madsen and G. Dennler, Sci. Rep., 2017, 7, 46630.

32 S. Wang, J. Yang, L. Wu, P. Wei, J. Yang, W. Zhang and Y. Grin, Chem. Mater., 2015, 27, 1071-1081.

33 Y. Qin, P. Qiu, R. Liu, Y. Li, F. Hao, T. Zhang, D. Ren, X. Shi and L. Chen, J. Mater. Chem. A, 2016, 4, 1277-1289.

34 S. M. Kauzlarich, S. R. Brown and G. J. Snyder, Dalton Trans., 2007, 21, 2099-2107.

35 S. Ray, A. Kumar, S. Majumdar, E. V. Sampathkumaran and D. D. Sarma, J. Phys.: Condens. Matter, 2001, 13, 607-616.

36 L. Fang, R. G. Iyer, G. Tan, D. J. West, S. Zhang and M. G. Kanatzidis, J. Am. Chem. Soc., 2014, 136, 11079-11084.
37 I. Shiozaki, M. Ohashi and H. Kadowaki, J. Phys. Soc. Jpn., 2000, 69, 3873-3877.

38 S. Bux, M. T. Yeung, E. S. Toberer, G. J. Snyder, R. B. Kaner and J. Fleurial, J. Mater. Chem., 2011, 21, 12259-12266.

39 B. Abeles, Phys. Rev., 1963, 131, 1906-1911.

40 G. A. Slack, Phys. Rev., 1957, 105, 829-831.

41 J. A. Callaway and H. C. Von Baeyer, Phys. Rev., 1960, 120, 1149-1154.

42 J. Yang, G. P. Meisner and L. Chen, Appl. Phys. Lett., 2004, 85, 1140-1142.

43 G. Zheng, X. Su, T. Liang, Q. Lu, Y. Yan, C. Uher and X. Tang, J. Mater. Chem. A, 2015, 3, 6603-6613. 